Variable range hopping conduction in N- and P-type in situ doped polycrystalline silicon nanowires

This article has been downloaded from IOPscience. Please scroll down to see the full text article.

2013 Semicond. Sci. Technol. 28025002

(http://iopscience.iop.org/0268-1242/28/2/025002)

View the table of contents for this issue, or go to the journal homepage for more

Download details:

IP Address: 82.227.205.211

The article was downloaded on 09/01/2013 at 20:18

Please note that terms and conditions apply. 


\title{
Variable range hopping conduction in $\mathrm{N}$ - and P-type in situ doped polycrystalline silicon nanowires
}

\author{
L Pichon, E Jacques, R Rogel, A C Salaun and F Demami \\ Institut d'Electronique et des Télécommunications de Rennes, Groupe Microélectronique, \\ UMR-CNRS 6164, Université de Rennes 1, Campus de Beaulieu, 263 avenue du général Leclerc, \\ 35042 Rennes cedex, France \\ E-mail: laurent.pichon@univ-rennes1.fr
}

Received 17 August 2012, in final form 23 November 2012

Published 27 December 2012

Online at stacks.iop.org/SST/28/025002

\begin{abstract}
Temperature dependence of electrical properties in N- and P-type in situ doped polycrystalline silicon nanowires synthesized by the sidewall spacer formation technique has been studied. Experimental analysis has been carried out for a temperature range from $200 \mathrm{~K}$ to $530 \mathrm{~K}$ on in situ doped polycrystalline silicon nanowires with doping level varying from $2 \times 10^{16}$ to $9 \times 10^{18} \mathrm{~cm}^{-3}$. Results show that for $\mathrm{N}$ - and P-type doped samples the temperature dependence of the conductivity follows the 3D variable range hopping model due to hopping between localized electronic states near the Fermi level. The corresponding densities of states are determined following exponential (tail states) distributions associated to the statistical shift of the Fermi level.
\end{abstract}

(Some figures may appear in colour only in the online journal)

\section{Introduction}

Quasi-one dimensional semiconductor nanowires feature a high surface-to-volume ratio and special electronic and optical properties, arising from the size confinement in the nano-sized channel region [1-4]. These unique aspects, especially in silicon nanowires (SiNWs), stimulate enormous research efforts to design and develop a new generation of high performance FET transistors and sensors by incorporating the SiNWs as the functional channels. The SiNWs technologies highlighting intrinsic compatibility to classical silicon technology have the potential to enable the development of innovative, high-performance and low-cost sensors applications, and bring significant impact in areas of electronics, mechanics, genomics and biomedical diagnosis.

SiNWs can be synthesized by the top-down approach, using various advanced methods such as e-beam [5], AFM [6] or deep UV [7] lithographies. The main disadvantage of these advanced lithographic tools with nanometer size resolution rests on their high cost. An alternative way is the bottom-up approach that usually employs metal catalytic growth. The most commonly used growth technique is the VLS (vapor-liquid-solid) method using metallic nanoparticles. The metal catalyst used is usually gold although other metals are also employed [8]. However, this approach suffers from the difficulty in precise positioning and self-orientation of the nanowires in a planar configuration.

In this work, SiNWs are synthesized without requiring costly lithographic tools, following the sidewall spacer formation technique (top down approach), commonly used in submicron scale device silicon technology to insulate device active area. The spacer at nanometric scale made of silicon constitutes the nanowire. This method is an alternative way to synthesize SiNWs in a $2 \mathrm{D}$ configuration $[9,10]$ compatible with planar Si technology. In this case, SiNWs present a polycrystalline structure, and their electrical behavior is expected to be different from crystalline SiNWs due to higher defect density. In this kind of disordered material, the electronic transport is strongly affected by the carriers trapping effects at these defects. The corresponding energy distribution of the states (DOS) is representative of the crystal quality of the material, and many studies have been devoted to the determination of the DOS. 

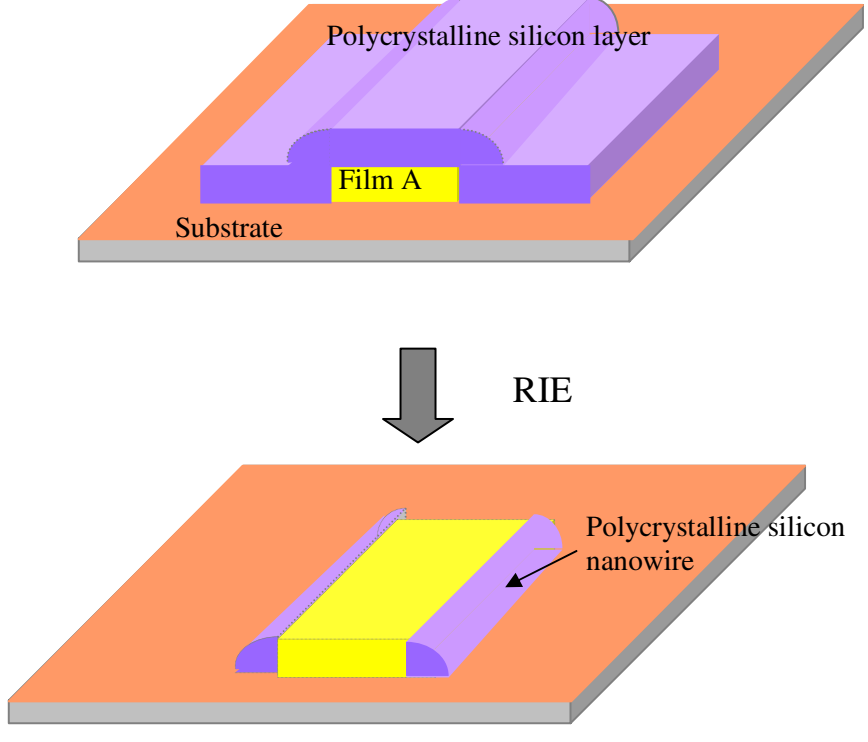

Figure 1. Fabrication of polycrystalline silicon NWs by the sidewall spacer formation technique.

Few studies about electrical behavior in polycrystalline silicon NWs have been reported. Thus, prior to developing polycrystalline silicon NWs based devices, in particular for high-performance electronics applications, a good understanding of electronic polycrystalline silicon NWs properties is required. In this paper, carrier transport in polycrystalline silicon NWs is analyzed as a function of temperature and doping level. Analysis is based on the hopping process between localized states related to the nanowire sizedependent defect density within the polycrystalline silicon nanowire. An experimental approach to extract exponential localized tail states distributions is presented.

\section{Experimental details}

The key fabrication steps of polycrystalline silicon NWs are illustrated in figure 1. First, a dielectric film A is deposited on an oxidized silicon substrate and patterned into islands by conventional UV lithography. Then, a polycrystalline silicon layer is deposited by the LPCVD technique. Accurate control of the polycrystalline silicon layer reactive ion etching (RIE) rate leads to the formation of nanometric size sidewall spacers that can be used as nanowires. The feasibility of this technological step was previously reported [10]. Thus, polycrystalline silicon NWs with a $50 \mathrm{~nm}$ curvature radius were synthesized (see figure $2(a)$ ). This method allows the fabrication of the parallel SiNWs network.

Phosphorus (or Boron) in situ doped polycrystalline silicon films used for nanowires were deposited by thermal decomposition of a mixture of pure silane and phosphine (or diborane) diluted in helium. The in situ doping level is controlled by adjusting the $\mathrm{PH}_{3} / \mathrm{SiH}_{4}$ (or $\mathrm{B}_{2} \mathrm{H}_{6} / \mathrm{SiH}_{4}$ ) mole ratio varying from 0 for undoped films to $6 \times 10^{-5}$ (or $10^{-4}$ ) for heavily doped films. The corresponding incorporated phosphorus (boron) atoms' concentrations, $C_{\mathrm{P}}\left(C_{\mathrm{B}}\right)$, previously determined from SIMS (secondary ions mass spectroscopy) analysis, varies from $2 \times 10^{16}$ to

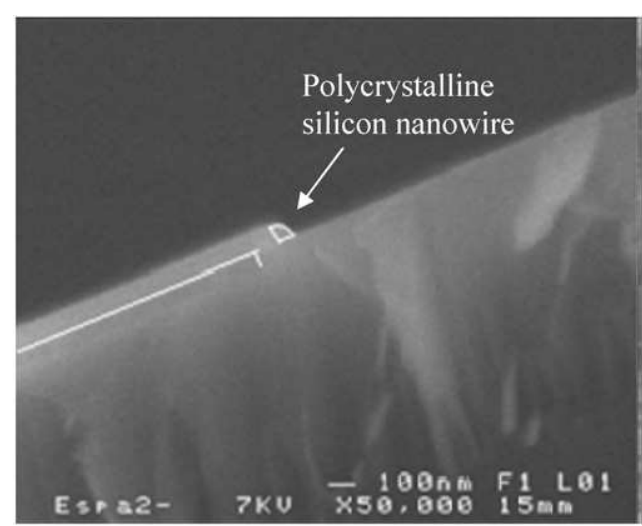

(a)

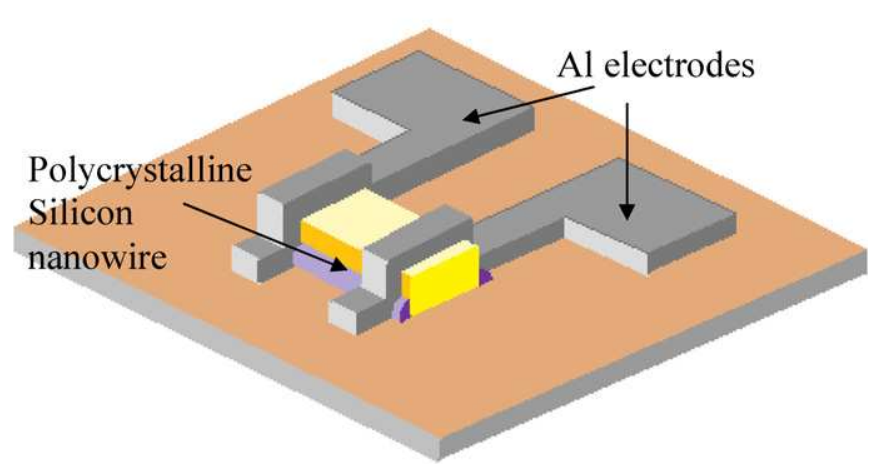

(b)

Figure 2. (a) SEM picture of the $50 \mathrm{~nm}$ radius curvature polycrystalline silicon NW cross section view. (b) Polycrystalline silicon NWs-based resistor used for electrical measurements.

$9 \times 10^{18} \mathrm{~cm}^{-3}$ (or $2 \times 10^{16}$ to $\left.6 \times 10^{18} \mathrm{~cm}^{-3}\right)$ [11]. Silicon films were deposited in an amorphous state at $550^{\circ} \mathrm{C}$ and $90 \mathrm{~Pa}$, and then crystallized by a thermal annealing in vacuum at $600{ }^{\circ} \mathrm{C}$ for $12 \mathrm{~h}$.

The in situ doped polycrystalline silicon NWs were integrated into resistors devices in coplanar structure (figure 2(b)) for electrical characterization. In this way, the nanowires were capped by a $\mathrm{SiO}_{2}$ layer deposited by the atmospheric pressure chemical vapor deposition (APCVD) technique at $420{ }^{\circ} \mathrm{C}$ and contact openings wet etched. Contact electrodes were made of thermally evaporated aluminum and defined by wet etching. Finally a thermal annealing in the forming gas $\left(\mathrm{N}_{2} / \mathrm{H}_{2}: 0.9\right)$ was carried out at $390{ }^{\circ} \mathrm{C}$ to ensure good electrical contacts. Resistors were fabricated with $10 \mu \mathrm{m}$ length parallel SiNWs.

Electrical dark conductivity of the polysilicon nanowires, $\sigma$, was deduced from $I(V)$ measurements collected at room temperature using a HP $4155 \mathrm{~B}$ semiconductor parameter analyzer. For current-temperature measurements, in the range $200 \mathrm{~K}$ to $530 \mathrm{~K}$, samples were placed in a cryostat in vacuum $\left(10^{-3}-10^{-4} \mathrm{~Pa}\right)$ and dark current was measured using a Keithley 617 electrometer.

\section{Results and discussion}

The polycrystalline silicon NWs structural quality is quite different from the polycrystalline silicon layer. Indeed, because the crystallization process of the amorphous silicon layer from 


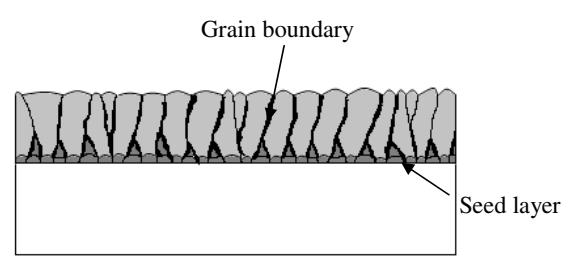

Columnar type structure of the polycrystalline silicon
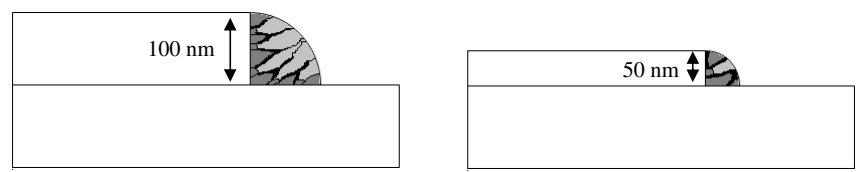

Polycrystalline silicon nanowires

Figure 3. Schematic illustration of structural defects in the core of the polycrystalline silicon NWs.

which NWs are processed starts from seeds located at the bottom interface (seed layer in figure 3 ), the polycrystalline silicon layer is usually considered to be made of large size $(>100 \mathrm{~nm})$ monocrystalline grains with a columnartype structure. In this case, according to Seto's theory [12] carriers transport is strongly affected by intergranular barriers induced by the carriers trapping effect at defects located at these grain boundaries. Such electrical behavior was previously reported by our group for $100 \mathrm{~nm}$ radius curvature polycrystalline silicon nanowires [13]. Indeed, as illustrated in figure 3 , the structural defect density involved (including grain boundaries) is higher in the inferior part of the layer (few nanometers thick) constituting the nanowires. Thus, the structural quality of the $50 \mathrm{~nm}$ radius curvature NWs is likely quite similar to micro- or nano-crystalline silicon material embedding quasi uniformly distributed high defect density (strongly affecting carriers transport). Therefore, a different transport mechanism is expected in these NWs. The most convenient model describing electrical properties in such disordered N- or P-type semiconductor material is the carrier hopping between localized states close to the conduction band $\left(E_{C}\right)$ or valence band $\left(E_{V}\right)$ edges, respectively, firstly proposed by Mott [14]. In this case, the carrier transport mechanism is thermally activated. The activation energy was deduced from the slope of the linear decrease of the electrical conductivity in an Arrhenius representation. For N- and P-doped polycrystalline silicon nanowires, the corresponding measured activation energy $\left(E_{A}\right)$ decreases with the doping concentration, and is significantly lower than the expected values for polycrystalline silicon layers [12] (figure 4(a). In particular, measured activation energy for undoped silicon nanowires is significantly lower than the expected midgap value for undoped silicon $(\sim 0.55 \mathrm{eV})$. Such values of $E_{A}$ highlight that thermoelectronic conduction is not predominant and validate the contribution of localized states for carrier transport. In addition, the decrease of $E_{A}$ is related to the increase of the carrier number in the silicon nanowires and thus also to the statistical shift of the Fermi level into the bandgap energy.

Electrical properties of polycrystalline silicon NWs were investigated as a function of temperature and in situ doping level. For all studied polycrystalline NWs, dark conductivity

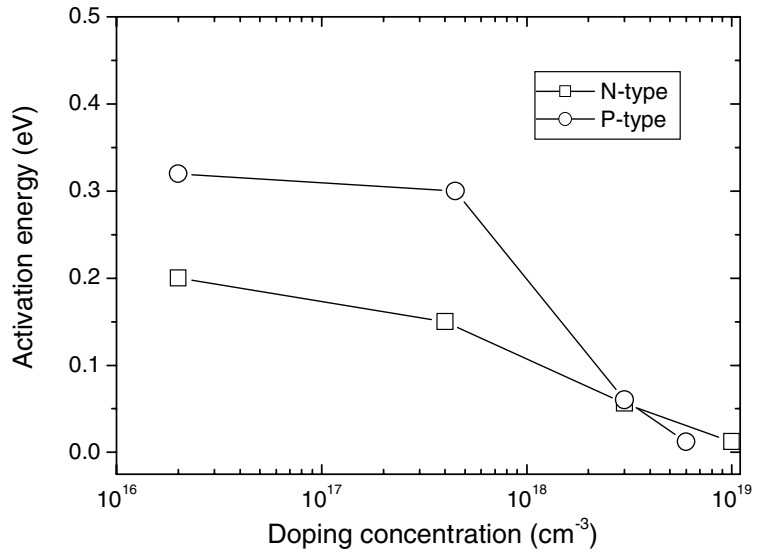

(a)

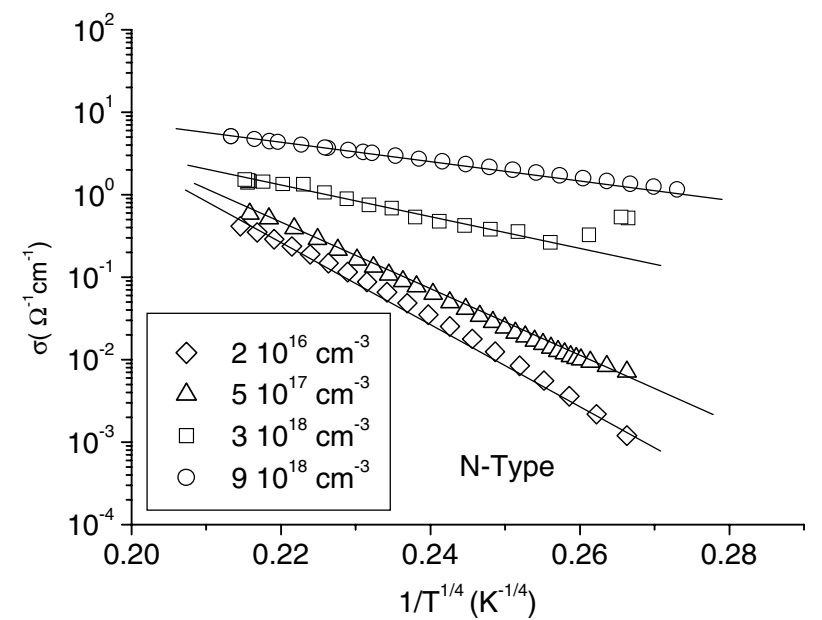

(b)

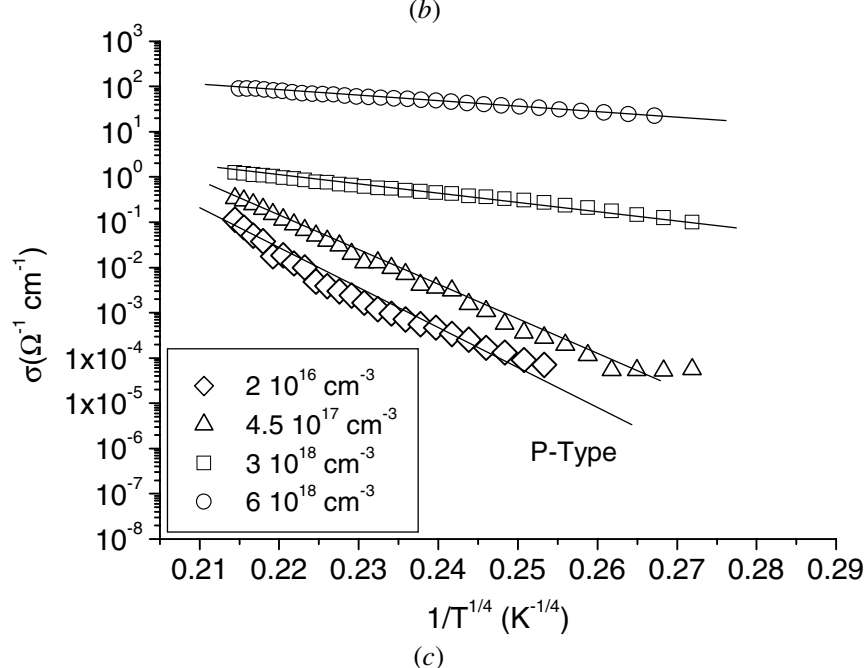

Figure 4. (a) Plots of the N-and P-doped polycrystalline silicon nanowires electrical conductivity activation energies versus doping concentrations. $(b)$ Dark conductivity as a function of $T^{-1 / 4}$ for N-type (a) and P-type (c) polycrystalline silicon NWs.

is thermally activated, and the temperature dependence, displayed in figures $4(b)$ and $(c)$, follows the $3 \mathrm{D}$ variable range hopping (VRH) mechanism described by Mott's law [14]:

$$
\sigma=\sigma_{0} \exp \left[-\left(\frac{T_{0}}{T}\right)^{1 / 4}\right]
$$


where in our case $\sigma_{0}$ and $T_{0}$ are both dependent on the doping level, and [15-19]:

$$
T_{0}=\frac{\gamma^{3} c^{4}}{k N\left(E_{F}\right)}
$$

with $k$ being the Boltzmann constant, $N\left(E_{F}\right)$ being the density of localized states near the Fermi level calculated by adjusting the parameter electronic wave decay length $\gamma^{-1}$ for localized states $\left(0.3 \mathrm{~nm} \leqslant \gamma^{-1} \leqslant 3 \mathrm{~nm}\right)$ and $c$ being a constant in the range of 2.06-4.2 depending on the $N\left(E_{F}\right)$ feature [16]. In this model, hopping refers to carrier tunneling transitions from occupied to unoccupied localized states, the state energy difference being bridged by emission of absorption of one or several phonons. The hopping between states close in energy level (even if they are wider spaced) becomes preferable to those between the nearest neighbors whose energies differ substantially. In addition, according to Mott [14], $N\left(E_{F}\right)$ is assumed as an energy-independent distribution, and a large number of previous studies on carrier transport [15-19], in particular in amorphous, micro- or nano-crystalline silicon layers, reported the average value depending on the quality of the material.

The conductivity prefactor, $\sigma_{0}$, deduced from linear fits in figures $4(b)$ and $(c)$, follows (figure 5)

$$
\sigma_{0}=\sigma_{0 m} \exp \left[\left(\frac{T_{0}}{T_{m}}\right)^{1 / 4}\right]
$$

where $T_{m}$ and $\sigma_{0 m}$ are independent of doping level, with $T_{m} \approx 755 \mathrm{~K}, \sigma_{0 m} \approx 3.4 \Omega^{-1} \mathrm{~cm}^{-1}$ for N-type NWs, and $T_{m} \approx 830 \mathrm{~K}, \sigma_{0 m} \approx 15.5 \Omega^{-1} \mathrm{~cm}^{-1}$ for P-type NWs. According to previous study based on numerical modeling of singlephonon hopping transport [16], such positive correlation between these two parameters predicts an exponential distribution of the localized electronic states $N\left(E_{F}\right)$ related to strained bond-type defects (tail states) in the core of the disordered semiconductor. Such a state distribution feature acts as a quality factor of the polycrystalline silicon NWs.

The localized states densities were deduced from relation (2) for each doping level for $\mathrm{N}$ - and P-type samples, respectively. Calculations were carried out for $c=4.2$ corresponding to an exponential state distribution [16]. The variations of $N\left(E_{F}\right)$ with doping level are reported in figure 6 for $0.3 \mathrm{~nm} \leqslant \gamma^{-1} \leqslant 3 \mathrm{~nm}$ (gray area). Previous works [20,21] reported that the doping species mainly affect the deep state defect distribution (dangling bonds) feature, in particular for hydrogenated amorphous silicon layer, but few changes for shallow tail states distributions. In this case, one can consider that the variations of $N\left(E_{F}\right)$ with the doping level should give an insight into the corresponding exponential states distributions for one adjusted $\gamma^{-1}$ value. However, representation of such DOS versus the statistical shift of the Fermi level into the bandgap energy is commonly used. In this way, one can assume that the average free electron $(n)$ and hole $(p)$ concentrations for N-type and P-type samples, respectively, are given by $n=$ $N_{C} \exp \left(-\left(E_{C}-E_{F}\right) k T\right)=\eta_{\mathrm{N}} C_{\mathrm{P}}$ and $p=N_{V} \exp \left(-\left(E_{F^{-}}-E_{V}\right) k T\right)$ $=\eta_{\mathrm{P}} C_{\mathrm{B}}$, where $\eta_{\mathrm{N}}$ and $\eta_{\mathrm{P}}(\leqslant 1)$ are the doping efficiencies, $N_{C}$ and $N_{V}$ the effective conduction and valence band state densities $\left(N_{C} \approx N_{V} \approx 3 \times 10^{19} \mathrm{~cm}^{-3}\right)$. Thus, the plots in figure 6 are directly related to the usual representations of

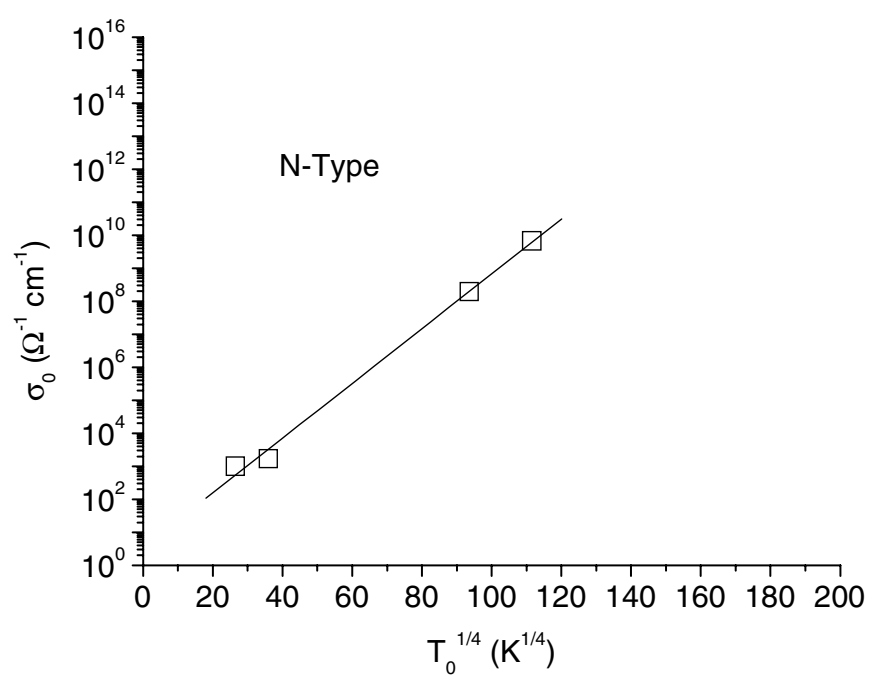

(a)

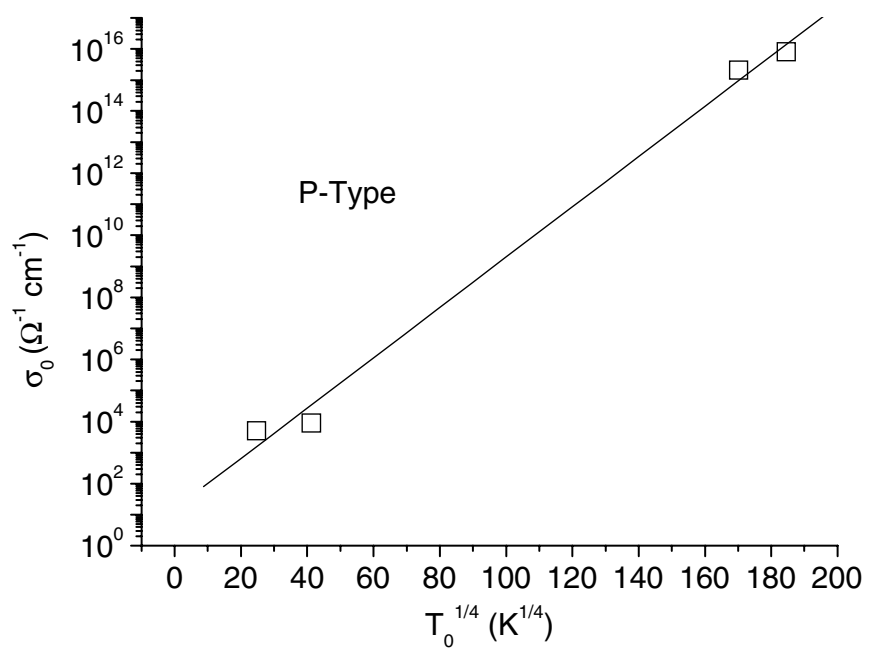

(b)

Figure 5. Dark conductivity prefactor as a function of $T_{0}^{1 / 4}$ for N-type $(a)$ and P-type (b) polycrystalline silicon NWs.

the DOS. Previous work [11] reported that for polycrystalline silicon layers deposited under the same conditions as for the nanowires, doping efficiency is high $(\sim 100 \%)$ for high doping levels. For such a doping efficiency for our polycrystalline silicon NWs, and such that it does not significantly influence the calculation of the Fermi level from the bandgap edges, $E_{F}-E_{C} \approx \mathrm{kT} \times \ln \left(C_{\mathrm{P}} / N_{C}\right)$ and $E_{V}-E_{F} \approx \mathrm{kT} \times \ln \left(C_{\mathrm{B}} / N_{V}\right)$ for $N$ - and $P$-doped NWs, respectively. In this case, the corresponding DOS versus $E_{F}$ were replotted in figure 7 for highest $\gamma^{-1}$ values to be consistent with $N\left(E_{F}\right)$ values obtained by others experimental methods for highly disordered silicon films [17, 20, 22].

According to these results, $N\left(E_{F}\right)$ related to shallow tail states distributions (close to the band gap edges) can be modeled as

$$
N\left(E_{F}\right)=N_{0 C} \exp \left(\frac{E_{F}-E_{C}}{E_{0 C}}\right)
$$

and

$$
N\left(E_{F}\right)=N_{0 V} \exp \left(\frac{E_{V}-E_{F}}{E_{0 V}}\right) .
$$




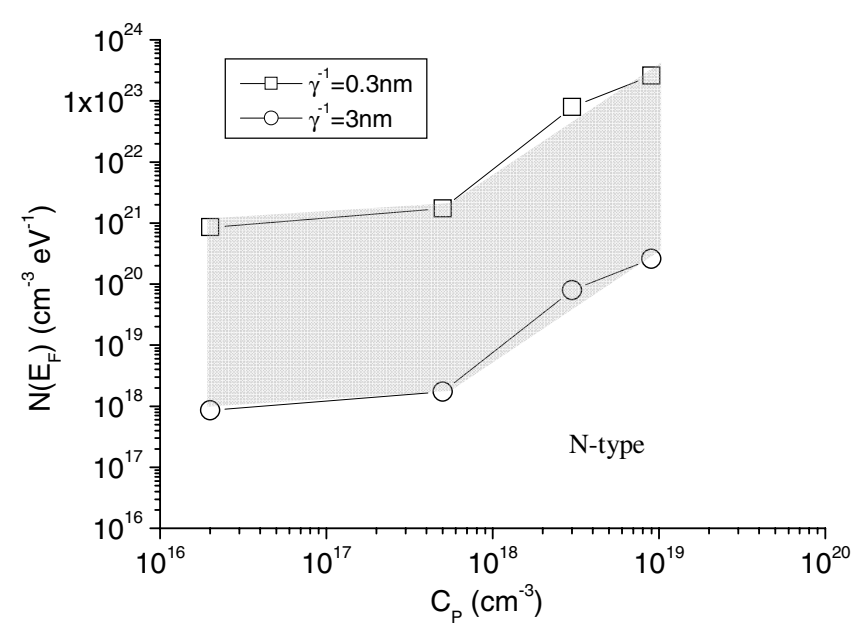

(a)

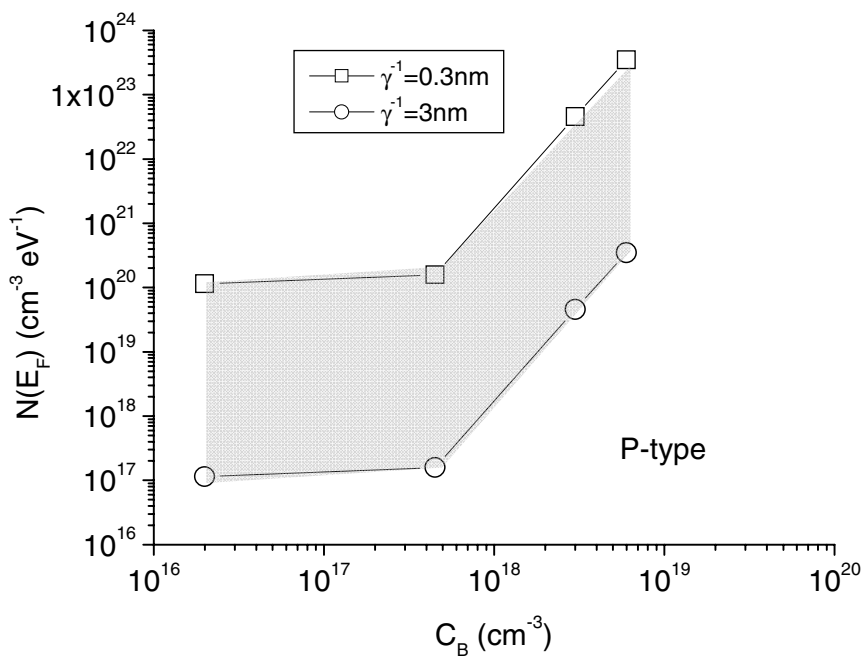

(b)

Figure 6. Distribution of localized states as a function of the doping level calculated with $c=4.2$, and for $0.3 \mathrm{~nm} \leqslant \gamma^{-1} \leqslant 3 \mathrm{~nm}$ (gray area), for N-type (a) and P-type (b) polycrystalline silicon NWs.

Here $N_{0 C}=3 \times 10^{21} \mathrm{~cm}^{-3} \mathrm{eV}^{-1}, E_{0 C}=15 \mathrm{meV}, N_{0 V}=$ $4 \times 10^{22} \mathrm{~cm}^{-3} \mathrm{eV}^{-1}$, and $E_{0 V}=9 \mathrm{meV}$ deduced from the linear fit of the plots in figure 7 corresponding to $N\left(E_{F}\right)$ values calculated for highly doped $\mathrm{N}$ - and P-type samples, respectively. Even if the $\gamma^{-1}$ value is not obvious, these results of our proposed bandgap shallow tail state model mean that $\gamma^{-1} \sim 3 \mathrm{~nm}$ is an appropriate value for the studied polycrystalline silicon NWs. Moreover, such a value has been recently reported for such $N\left(E_{F}\right)$ behavior deduced from analysis of the field effect conductance of low-temperature $\left(\leqslant 600^{\circ} \mathrm{C}\right) \mathrm{N}$-channel polycrystalline silicon TFTs reporting experimental determination of shallow tail state distribution close to the conduction band [23]. In addition, these exponential states distributions are consistent with theoretical studies previously reported in the case of hydrogenated amorphous silicon [20, 21]. However, for lightly doped samples the doping efficiency is significantly lower than for highly doped ones. Therefore, the plots of $N\left(E_{F}\right)$ are not representative of the tail state distributions following (4) and (5). In this case, due to the high defect density

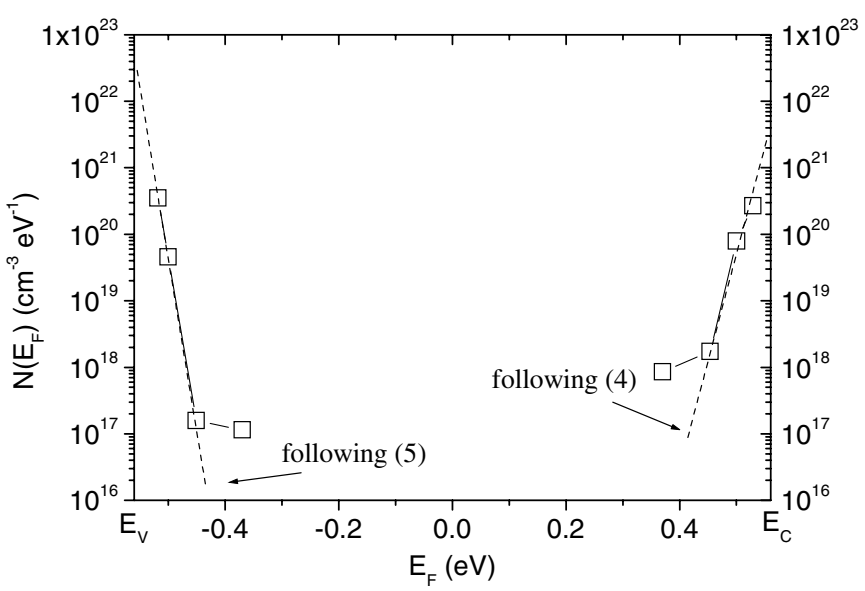

Figure 7. Distributions of localized states calculated with $\gamma^{-1}=$ $3 \mathrm{~nm}$ as a function of the position of the Fermi level. The energy bandgap value is $E_{G}=1.12 \mathrm{eV}$. Dotted plots: calculated exponential shallow tails states distributions (close the bandgap edges) following (4) and (5) with a linear regression coefficient $R=0.97683$ or 0.99999 , respectively.

within the polycrystalline silicon NWs one can consider that the Fermi level is pinned and thus a quasi constant value of $N\left(E_{F}\right)$ is measured. This result could be related to the energy-independent $N\left(E_{F}\right)$ assumed in Mott's model, usually reported in many previous experimental analysis of the 3D VRH mechanism in undoped disordered semiconductors [15, 17-19, 24]. Only theoretical or numerical models proposed an exponential distribution of localized states for hopping process $[16,25]$. Therefore, our experimental analysis gives an approach to determine the DOS to qualify disordered semiconductors and validates the model of exponential states distribution for the VRH model.

\section{Conclusion}

Analysis of the dark conductivity of $50 \mathrm{~nm}$ curvature radius $\mathrm{P}$ - and $\mathrm{N}$ - type in situ doped polycrystalline silicon nanowires shows that carrier transport is described by a $3 \mathrm{D}$ variable range hopping mechanism between defect states near the Fermi level. In this experimental study, we emphasized the influence of the structural defect densities on specific description of carriers transport. Analysis shows that the localized state distributions are related to the statistical shift of the Fermi level. In addition, our study allows the determinations from the VRH model of the corresponding exponential shallow tail state distributions close to the valence and conduction bands' edges to qualify the polycrystalline silicon nanowires.

\section{Acknowledgments}

This work was supported by the Agence Nationale de la Recherche (France) with the Project Sena (ANR-09JCJC0072-01). 


\section{References}

[1] Cui Y et al 2001 Science 2931289

[2] Hsu J F et al 2005 Japan J. Appl. Phys 442626 Part 1-Regular Papers Brief Communications \& Review Papers

[3] Shao M-W, Shan Y-Y, Wong N-B and Lee S-T $2005 A d v$. Funct. Mater. 151478

[4] Roy S and Gao Z 2009 Nano Today 4318

[5] Li Z, chen Y, Li X, Kamins TI, Nauka K and Williams R S 2004 Nanoletters 4245

[6] Ionica I, Montes L, Ferraton S, Zimmermann J, Saminadayar L and Bouchiat V 2005 Solid State Electron. 491497

[7] Yang L, Lee D H, Chen H Y, Chang C Y, Liu S D and Huang C C 2004 VLSI Symp. Tech. Dig. p 196

[8] Wang Y, Schmidt V, Senz S and Gösele U 2006 Nature Nanotechnol. 1 186-9

[9] Hsu H H, Liu T W, Chan L, Lin C D, Huang T Y and Lin H C 2008 IEEE Trans. Elect. Dev. 553063

[10] Demami F, Pichon L, Rogel R and Salaun A C 2009 Mater. Sci. Eng. 6012014

[11] Briand D, Sarret M, Le Bihan F, Bonnaud O and Pichon L 1995 Mater. Sci. Tech. 111207
Briand D 1995 PhD Dissertation Université de Rennes 1, France

[12] Seto J W 1975 J. Appl. Phys. 465247

[13] Demami F, Rogel R, Salaun A C and Pichon L 2011 Phys. Status. Solidi C 8827

[14] Mott N F 1969 Phil. Mag. 19835

[15] Paul D K and Mitra S S 1973 Phys. Rev. Lett. 131000

[16] Godet C 2002 J. Non-Cryst. Solids 299-302 333

[17] Concari S B, Buitrago R H, Gutierrez M T and Gandia J 2003 J. Appl. Phys. 942417

[18] Concari S B and Buitrago R H 2004 J. Non-Cryst. Solids 338-340 331

[19] Ambrosone G, Coscia U, Cassinese A, Barra M, Restello S, Rigato V and Ferrero S 2007 Thin Solid Films $\mathbf{5 1 5} 7629$

[20] Winer K 1989 Phys. Rev. Lett. 631487

[21] Roberton J 1984 J. Phys. C: Solid State Phys. 17 L349

[22] Schmidt M, Schoepke A, Korte L, Milch O and Fuhs W 2004 J. Non-Cryst. Solids 338-340 211

[23] Pichon L and Rogel R 2011 Appl. Phys. Lett. 99072106

[24] Thamilselvan M, Premnzeer K, Mangalaraj D and Nanrayandass Sa K 2003 Physica B 337404

[25] Godet C 2003 Diam. Relat. Mater 12159 